\title{
Study of Agar Proportions Effect on a Gamma Ray
} Synthesized Hydrogel

Nour Elhouda Ben Ammar ${ }^{*}$, Taib Saied ${ }^{2}$, Arbi Mejri ${ }^{3}$, Faouzi Hosni ${ }^{3}$, Adel Mnif ${ }^{1}$ and Ahmed Hichem Hamzaoui $^{1}$

1. Laboratory of Useful Materials Valuation, National Center for Research in Materials Sciences, Borj Cedria Technopark, BP 73, 8027 Soliman, Tunisia

2. Laboratory of Analytical Chemistry and Electrochemistry, University of Tunis el Manar, Science Campus, 2092 Tunis, Tunisia

3. Treatment Unit by Ionizing Radiation, National Center of Sciences and Nuclear Technology, Sidi Thabet Technopark, BP 72, 2020

Sidi Thabet, Tunisia

\begin{abstract}
In this study, we aimed to identify the effect of agar proportion on the synthesized superabsorbent properties based on PVP (Polyvinyl pyrrolidone), poly (ethylene glycol) (PEG) and agar. In a first stage, the study of swelling and deswelling behavior of synthesized hydrogel was done. It was proved that agar acts as a crosslinking inhibitor since it competes with free radicals. The $\mathrm{pH}$ versus swelling time was studied. The polymer was characterized by Fourier transform infrared spectra analysis and X-ray Diffraction. The removal of $\mathrm{Cu}(\mathrm{II})$ from aqueous solution by the prepared PVP/PEG/agar hydrogel was studied. Also, the influences of $\mathrm{pH}$ and contact time were investigated throughout adsorption experiments. The maximum retention capacity was found to be 36.5 $\mathrm{mg} \mathrm{g}^{-1}$ for the hydrogel containing $1 \%$ of agar. The kinetic parameters were calculated from the experimental data and it was shown that they fit well with pseudo-second-order kinetic model.
\end{abstract}

Key words: Hydrogel, PVP, copper, agar, gamma radiation.

\section{Introduction}

Hydrogels are three-dimensional polymeric networks whose main feature is the ability to absorb and retain large volumes of water. Because of their excellent characteristics, superabsorbents have been widely used in many areas, such as disposable diapers, feminine napkins [1], humidity controller in packaging [2], protect Optical Fiber Cables from water penetration [3], as agriculture support to maintain water in plants neighborhood [4] and because of their weak irritation potential [5] they are used in hygienic and medical products such as wound dressing [6]. These polymeric hydrogels are promising materials in the field of hydrometallurgical applications as well as water purification because of their chemical stability [7].

\footnotetext{
*Corresponding author: Nour Elhouda Ben Ammar, research
} assistant, research field: polymers.
These hydrogels can be obtained by simple crosslinking of two linear polymers and modified by blending some natural polymers. These hydrogels exhibit properties that are different from those of the initial polymers. This can be made by radical reaction introduced by various electromagnetic irradiations. JM. Rosiak and coworkers [8] used gamma irradiation to make hydrogel marketed under the name of AQUA-GEL ®. However, Z. Ajji and coworkers [6] used electron beam with the same composition in order to compare the quality. Similarly, C. Lilian and colleagues [9], have used the direct UV photocrosslinking to avoid the use of high energy radiation.

The present work reports the use of Gamma irradiation technique as a clean and environmentally friendly method, the properties of the obtained material will be compared in our next paper with those of a chemically synthesized hydrogel using free 
radical initiators such as ammonium persulfate. PVP is commonly used as a food additive considering its inoffensive properties, it was then selected as a base polymer for synthesizing a hydrogel for agricultural applications. Also the impact of agar proportion on the degree of crosslinking, and therefore on the swelling properties were studied. The hydrogel retention capacity with respect to $\mathrm{Cu}$ (II) ions, and the amount of metallic ions absorbed by the polymers was determined using an atomic absorption spectrophotometer, and finally the adsorption kinetics was determined.

\section{Experimental and method}

\subsection{Materials}

AlI reagents were commercially available and used without further purification. Reagents used in the synthesis of gamma irradiated crosslinked hydrogel are PVP (MW : 360,000) (Sigma), PEG (MW: 3,000) (Fluka), agar (Sigma Alderich biochimica), diluted solutions of $\mathrm{HCl}$ (Scharlau) and $\mathrm{NaOH}$ (Scharlau) in order to fix $\mathrm{pH}$, copper (II) contained in the $\left(\mathrm{CuSO}_{4} \cdot 5 \mathrm{H}_{2} \mathrm{O}\right)$ ( sigma) and distilled water.

\subsection{Synthesis of Hydrogel}

$8 \%$ of PVP (MW: 360,000 ), $5 \%$ of PEG (MW: $3,000)$, a proportion of $0.5,1,1.5$ and $2 \%$ of Agar were mixed in a ball, and adjusted to $100 \%$ with distilled water. The ball was heated under reflux device at temperature which exceeds $80{ }^{\circ} \mathrm{C}$ and was left under agitation until the solution becomes crystal clear [7]. Obtained solutions were transmitted into 40 $\mathrm{ml}$ closed plastic flasks in order to be irradiated with $\mathrm{Co}^{60} \gamma$-ray irradiation.

\subsection{Irradiation}

Irradiation of the hydrogel samples were done at the Tunisian semi-industrial $\mathrm{Co}^{60}$ gamma irradiation facility at the dose rate of about $1 \mathrm{kGy} \cdot \mathrm{h}^{-1}$ [10]. Dosimetry was done using Fricke chemical dosimeter and the traceability was established with alanine/EPR dosimetry system in terms of absorbed dose to water traceable to Aérial Secondary Standard Dosimetry Laboratory (SSDL), Strasbourg-France.

\subsection{Measurement}

FT-IR spectra of the polymers were recorded with Perkin Elmer FT-IR system in the range 4,000-600 $\mathrm{cm}^{-1}$. Atomic absorption data were obtained using AAS vario 6 spectrophotometer. X-rays diffraction of samples were done using a diffractometer model X'PERT Pro MPD PANALYTICAL. High-voltage generator: $60 \mathrm{KV}, 60 \mathrm{~mA}$. X-rays source: Ceramic tube of $\mathrm{X}$ - rays with copper anode of $1,8 \mathrm{~kW}$ power. Goniometer $\theta-2 \theta$ configuration: Radius of de goniometer: $240 \mathrm{~mm}$, fixed $\mathrm{RX}$ source, mobile detector $X^{\prime}$ Celerator $(2 \theta)$, mobile sample $(\theta)$ (in horizontal position ), step : $0.001^{\circ}$.

\subsection{Swelling Study}

Before starting swelling studies, all soluble parts contained in the hydrogel should be removed. Therefore, drying samples were firstly provided and then left in distilled water during several days, until reaching a constant weight corresponding to the adsorption balance. Final samples were then dried once more time at room temperature in order to be used for swelling studies.

The prepared gels were cut into discs and then swollen in buffer solutions having different $\mathrm{pH}$, ranging from 1 to 10.5 at $25{ }^{\circ} \mathrm{C}$. The swelling ratio was determined from the following equation:

$$
S=\left(\frac{w_{f}-w_{i}}{w_{i}}\right) * 100
$$

where, $\mathrm{w}_{\mathrm{f}}$ and $\mathrm{w}_{\mathrm{i}}$ are the weights of the swollen and the dry hydrogel, respectively.

\subsection{Isothermal Dehydration}

The dehydration behavior was followed by isothermal measuring, of the percentage mass decrease of the hydrogel, at room temperature [7]. This dehydration percentage was determined using 
following equation:

$$
\operatorname{Dehydration}(\%)=\left(\frac{w_{f}}{w_{i}}\right) * 100
$$

\subsection{Adsorption Experiment}

\subsubsection{PH versus the Metal Retention}

The $\mathrm{pH}$ versus the metal retention was established using $20 \mathrm{ml}$ of metal ion solution $\left(500 \mathrm{mg} \cdot \mathrm{L}^{-1}\right)$. The $\mathrm{pH}$ values were selected to be below 5 because beyond which, we observe the apparition of $\mathrm{Cu}(\mathrm{OH})_{2}$ precipitate [11]. $\mathrm{pH}$ of metal ions solution was adjusted by adding diluted $\mathrm{NaOH}$ or $\mathrm{HCl}$ solution. The adsorbed quantity of copper was determined using following equation:

$$
Q_{a d}=\left(C_{0}-C_{f}\right) * \frac{V}{W}
$$

where, $\mathrm{C}_{\mathrm{o}}$ and $\mathrm{C}_{\mathrm{f}}$ are the concentrations of metal ions before and after the adsorption, respectively $\left(\mathrm{mg} \cdot \mathrm{L}^{-1}\right), \mathrm{V}$ is the volume of solution $(\mathrm{L})$ and $\mathrm{W}$ is the weight of the hydrogel (g).

\subsubsection{Rate of Metal Ion Adsorption}

Batch studies were carried out with the prepared $\mathrm{PVP} / \mathrm{PEG} /$ agar hydrogel in order to determine the effect of retention duration of metal ion. $50 \mathrm{ml}$ of $\mathrm{Cu}$ (II) solution $(500 \mathrm{mg} \cdot \mathrm{L}-1)$ are mixed with prepared hydrogel and stirred. At regular time intervals, aliquots were taken from the solution and analyzed by atomic absorption spectroscopy.

\subsubsection{Kinetic Models}

Previous results were used to examine the effectiveness of the adsorption of various prepared hydrogels. For information on the different stages of adsorption over time, adsorption phenomena must be studied kinetically.

A simple kinetic analysis represented by a four different kinetic models was applied in this study to fit adsorption efficiency of the different prepared hydrogels. These are the pseudo-first-order equation, the pseudo-second-order equation, the equation of Elovich and intraparticules diffusion model. These models use the following linearized relationship [12].
Pseudo first-order: This is a widely used model. It assumes that the speed is proportional to the difference between the adsorbed amount at equilibrium "qe" and adsorbed amount " $\mathrm{q}_{\mathrm{t}}$ " at time $\mathrm{t}$ :

$$
\ln \left(q_{e}-q_{t}\right)=\ln q_{e}-k_{1} t
$$

Pseudo second-order [13] (Ho et al., 1998): This model is used more than the previous since it is valid for a wide range of time. It assumes heterogeneity of adsorption sites.

$$
\frac{t}{q}=\left(\frac{1}{k_{2} q_{e}^{2}}\right)+\frac{t}{q_{e}}
$$

The two previous models are based on the composition of the milieu

Elovich [14], this model assumes that the adsorption is through a chemisorption mechanism

$$
q_{t}=\beta \ln (\alpha \beta)+\beta \ln t
$$

Intraparticle diffusion model: This model is proposed by Weber and Morris [15]. It highlights the type of diffusion mechanism that is involved in the kinetics. The representation of the equation is very simple. The model equation is of the form:

$$
q_{e}=k_{i n t} t^{(1 / 2)}
$$

where, $\mathrm{q}_{\mathrm{e}}$ and $\mathrm{q}_{\mathrm{t}}$ are the amount of copper (II) ions adsorbed on the adsorbent at equilibrium and at various time $\left(\mathrm{mg} \cdot \mathrm{g}^{-1}\right), \mathrm{k}_{1}\left(\mathrm{~min}^{-1}\right)$ and $\mathrm{k}_{2}\left(\mathrm{~g} \cdot \mathrm{mg}^{-1} \cdot \mathrm{min}^{-1}\right)$ are the rate constant of the pseudo first-order and pseudo second-order model respectively, $\alpha$ is the initial adsorption rate $\left(\mathrm{mg} \cdot \mathrm{g}^{-1} \cdot \mathrm{min}^{-1}\right)$ and $\beta$ is desorption constant $\left(\mathrm{g} \cdot \mathrm{mg}^{-1}\right)$ for Elovich model and $\mathrm{k}_{\text {int }}$ is the intraparticle diffusion constant $\left(\mathrm{mg} \cdot \mathrm{g}^{-1} \cdot \mathrm{min}^{-1 / 2}\right)$.

The two previous models assume that the adsorption is through the mass transfer mechanism by diffusion.

\section{Results and Discussion}

\subsection{Synthesis and Mechanism Aspects}

Radiation-induced crosslinking polymerization has 
long been recognized as a powerful technique for the formation of hydrogels with unique properties. Here, ionizing radiation was used for the preparation of PVP/PEG/agar hydrogel. For the synthesis of desired hydrogel by $\gamma$-ray irradiation, a possible mechanism (involving more important steps) is proposed in scheme 1. It should be mentioned that during the irradiation of PVP, PEG agar and water mixture, most of the energy is absorbed by water and only a very small fraction by other components. [16]. As a result, short-lived reactive species are formed such as $\mathrm{OH}$ radicals and $\mathrm{H}$-atoms (reaction $\mathrm{A}$ ).

Then polymer radicals could be generated due to the abstraction of hydrogen atoms from macromolecules by hydroxyl radicals and hydrogen atoms (reaction B).

Davis et al [17] were the first to propose the structure of poly (N-vinyl-2-pyrrolidone) radicals generated during irradiation of PVP in aqueous solution.

$$
\begin{array}{cc}
\mathrm{H}_{2} \mathrm{O} \stackrel{\text { rray }}{\longrightarrow} \dot{O H}+\dot{H} & \text { (Reaction A) } \\
\text { Macromolecule }+\dot{\mathrm{OH}}(\dot{\mathrm{H}}) \rightarrow \text { Macroradical }+\mathrm{H}_{2} \mathrm{O}\left(\mathrm{H}_{2}\right) & \text { (Reaction B) }
\end{array}
$$

The most probable dominant radical structure are 1 and 2 illustrated in scheme 1 . They are formed by the abstraction of hydrogen from the methylene and methylidene groups. The third one is formed by the hydrogen abstraction from terminal hydroxide (it is important to ad 3 on scheme 1).

During the irradiation of aqueous solution, generated macroradicals may react in different ways; either one radical (chain scission and hydrogen transfer) or two-radical (cross-linking and disproportionation). It may be also a recombination that may occur between two radicals localized in the same chain or between two radicals within separate macromolecules.

It should be noticed that controlling the proportion of recombination and disproportionation reactions is set by the radical structure and it might be very difficult to control this parameter.

Hydrogels synthesis upon irradiation of polymer in solution may occur basically by intramolecular reactions and intermolecular cross-linking of polymer radicals. Since there is always a competition between these two processes and degradation, the ultimate goal is to choose the right conditions that promote intramolecular and intermolecular cross-linking and reduce the probability of undesirable processes.

The combination of these processes may form PVP/PEG/agar hydrogel with a SIPN structure (semi interpenetrating network) whereby agar and PEG are entangled within the crosslinked PVP or a grafted network whereby agar and/or PEG is grafted into the PVP backbone [18]. The effect of the network structure could influence the swelling behavior of the $\mathrm{PVP} / \mathrm{PEG} /$ agar hydrogel. This is clearly demonstrated in Figs 3 and 4.

\subsection{Characterization}

In order to identify the variation of chemical structure of the crosslinked hydrogel, Infrared spectroscopy technique was used. Fig. 1 illustrates the characteristic absorption bands of synthesized hydrogel, PVP, PEG, and agar. The FTIR spectra of PVP show a distinct carbonyl peak around $1,639 \mathrm{~cm}^{-1}$ that persists in the crosslinked hydrogel but shifted to $1,645 \mathrm{~cm}^{-1}$ witch suggested strong hydrogen bonding interaction between $\mathrm{C}=\mathrm{O}$ groups and $\mathrm{OH}$ of agar or terminal $\mathrm{OH}$ of PEG which could confirm that they are physically entangled within the crosslinked polymer [19]. The intensity of characteristic adsorption bands of PVP centered at 2,954 $\mathrm{cm}^{-1}$ corresponding to the $\mathrm{C}-\mathrm{H}$ stretching increases showing a possible superposition of different PVP linear chains together to form crosslinked PVP, and bands at 1,494, 1,462, 1,423 and 1,289 $\mathrm{cm}^{-1}(\mathrm{C}-\mathrm{N})$ also appeared in the spectrum of synthesized hydrogel. This indicates that linear PVP polymer chains exist in 


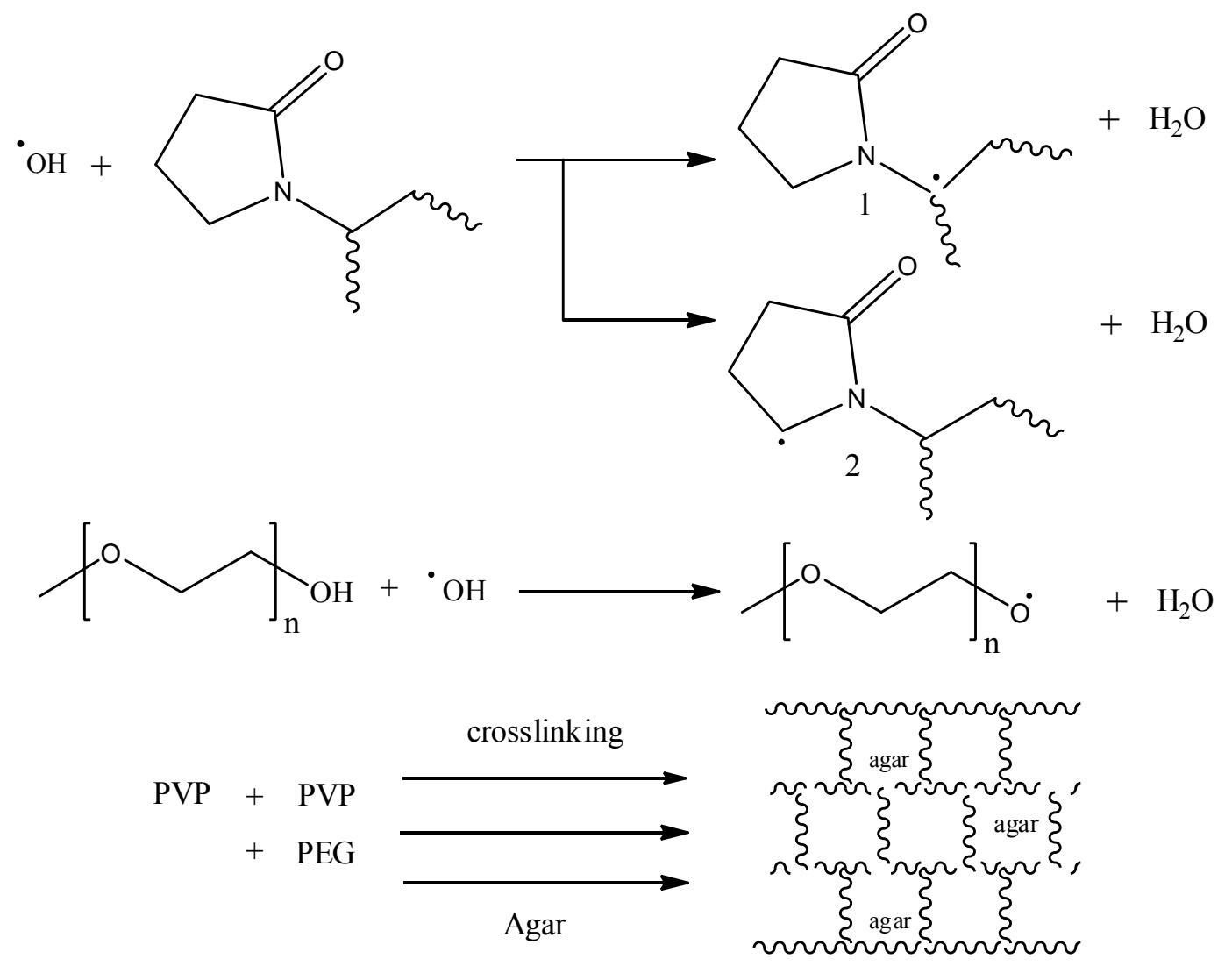

Scheme 1

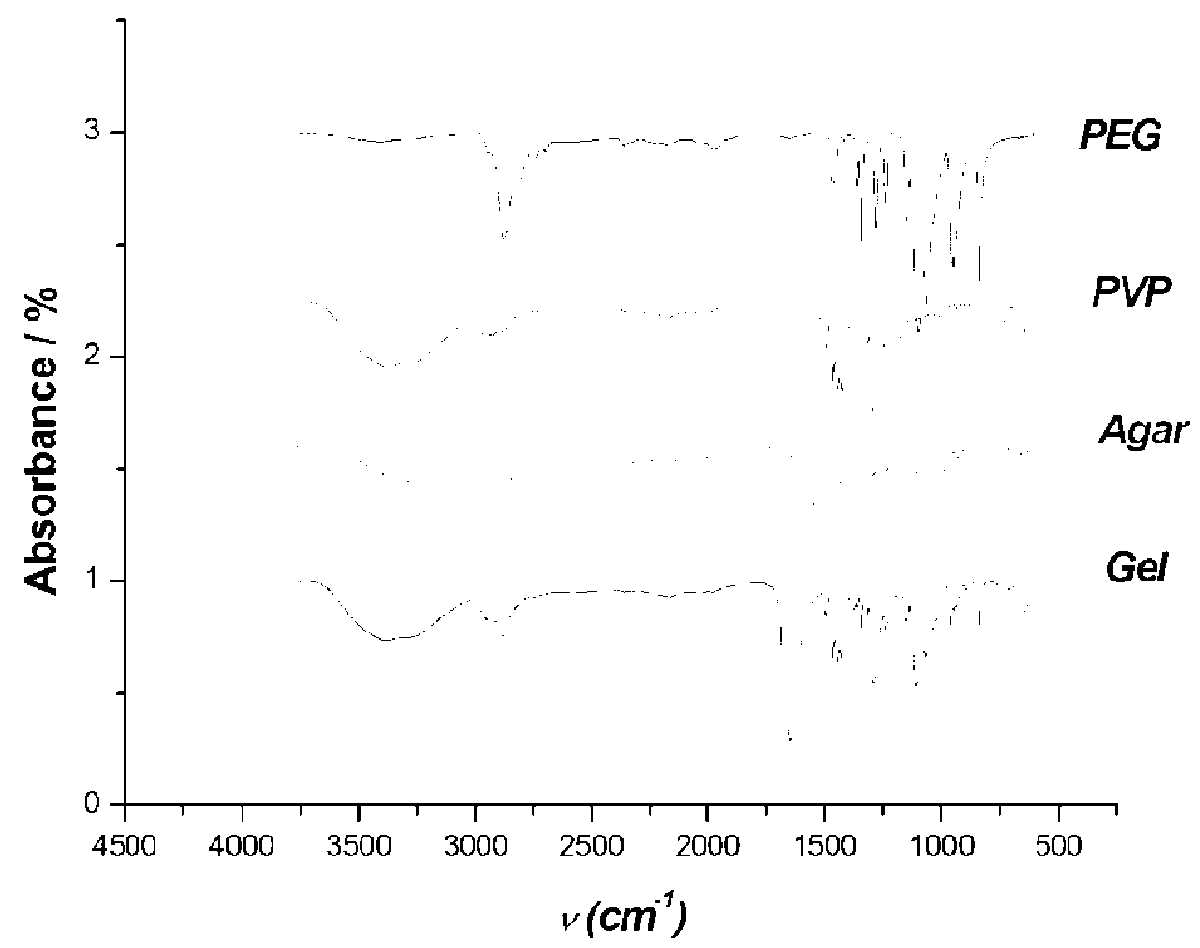

Fig. 1 FTIR of PVP, PEG Agar and synthesized hydrogel (1\% of agar after irradiation at $50 \mathrm{kGy}$ ). 
the network of hydrogel and are combined with agar and PEG by hydrogen-bonding interaction [20] (Wang et al., 2010) .

PEG characteristics absorption bands, between $1,040 \mathrm{~cm}^{-1}$ and $1,200 \mathrm{~cm}^{-1}$, related to stretching vibration $\mathrm{C}-\mathrm{O}$ and $\mathrm{C}-\mathrm{O}-\mathrm{C}$, appear in the hydrogel showing that they are not concerned by the crosslinking reaction as it is in the case of terminal $\mathrm{OH}$ groups, in addition, because their characteristic absorption band at $2881 \mathrm{~cm}-1$ persists. And then, as shown in previous works [21]. PEG acts mainly as a plasticizer, thereby increasing the distance between the molecules of PVP and does not participate in the crosslinking reaction.

The combination of the above observations leads to the conclusion that no grafting has been produced between the frameworks of the agar, PEG and PVP since there is no transformation in the spectrum of the hydrogel. No appearance of new groups and new functions, but only the displacement of existing bands due to the change in electronic environment and increase in intensity of other groups due to crosslinking phenomena. As illustrated in scheme 1, agar was physically entangled within the network of crosslinked PVP which is confirmed by FTIR analyses since there is only hydrogen bonding between PVP, PEG and agar and then it confirms an interpenetrating network structure.

X-ray diffraction of pure PVP, PEG and synthesized hydrogel are presented in Fig. 2, numerous diffraction peaks of synthesized hydrogel indicate the crystallinity presence. PVP shows an amorphous structure, while pure PEG displays two peaks with high intensity at $2 \theta$ respectively 19.3 and 23.4, beside other peaks at $2 \theta$ equal to 13.63 and 26.9. After the irradiation of PVP, PEG and agar mixture, the strong reflection of PEG became weaker and decreased to almost $84 \%$. This phenomenon could be explained as follows: in the crosslinked hydrogel, PEG strongly interacted with hydroxide radicals and agar, consequently, such complexation destroyed the organized arrangement of PEG [22]. The diffraction

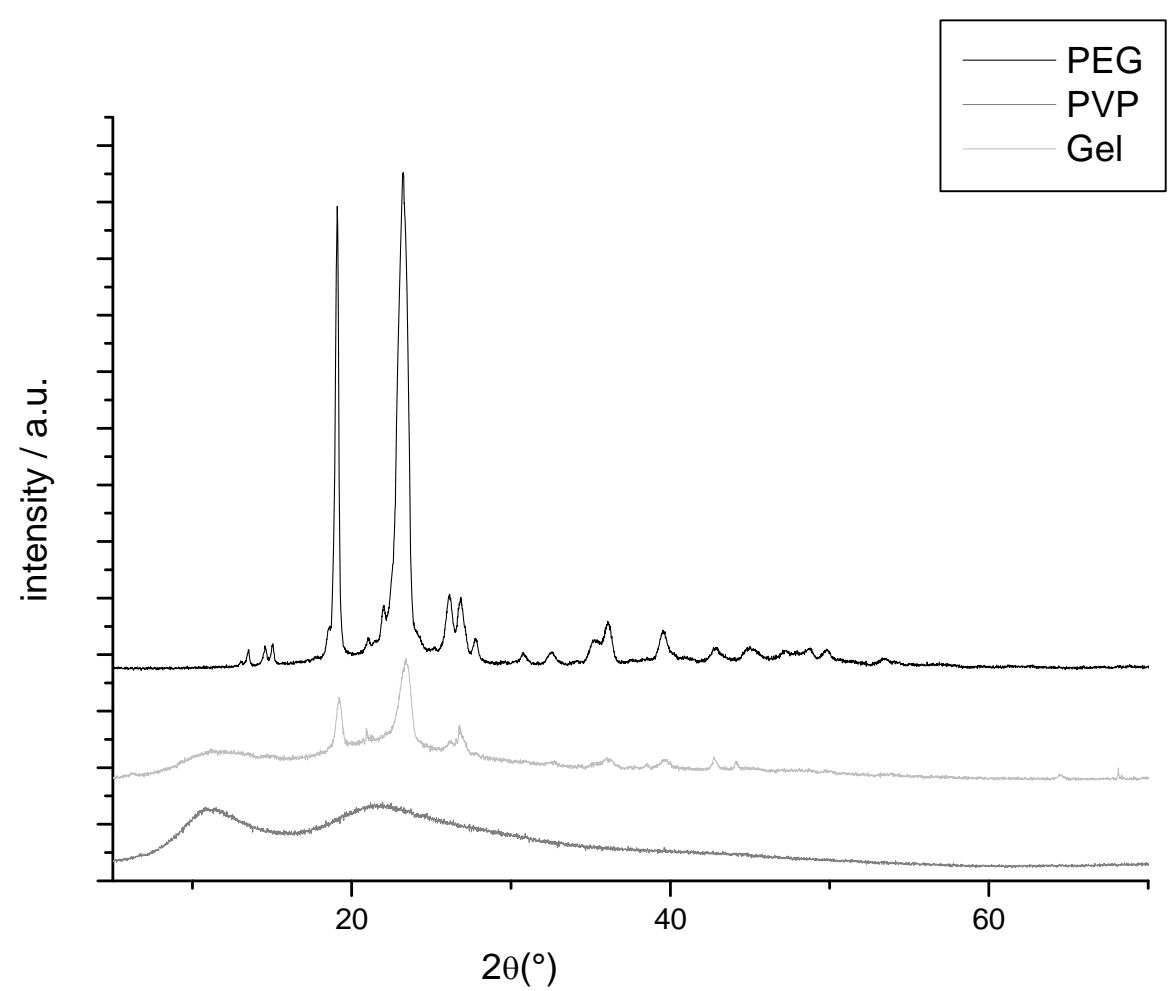

Fig. 2 X-ray diffraction of pure PVP, PEG and synthesized hydrogel. 
peak of PEG at $2 \theta=13.63^{\circ}$ and $32.6^{\circ}$ disappeared and some new superposition peaks were formed. This result suggested the formation of 3D-network within the crosslinked hydrogel.

\subsection{Swelling and Deswelling Study}

After synthesis of four hydrogel samples containing $0.5,1,1.5$ and $2 \%$ of agar, the effect of $\mathrm{pH}(1-10.5)$ on the swelling behavior of the prepared hydrogels was investigated. Fig. 3 shows that synthesized polymers have a very important swelling capacity in water for all the studied $\mathrm{pH}$ range. Like that is represented, the maximum of hydration of all the hydrogels is obtained at $\mathrm{pH}$ between 6 and 7 , excepted for the case $0.5 \%$ of Agar which reaches its maximum of swelling at $\mathrm{pH}$ of 8.5 , and for which the swelling is strongly influenced by the $\mathrm{pH}$.

As illustrated in Fig. 3, blending PVP/PEG with 0,5 to $1 \%$ of agar and irradiating the blend can improve the water retention capacity to as much as $1,650 \%-2,250 \%$. This may be explained by the structure of Agar itself because it contains the

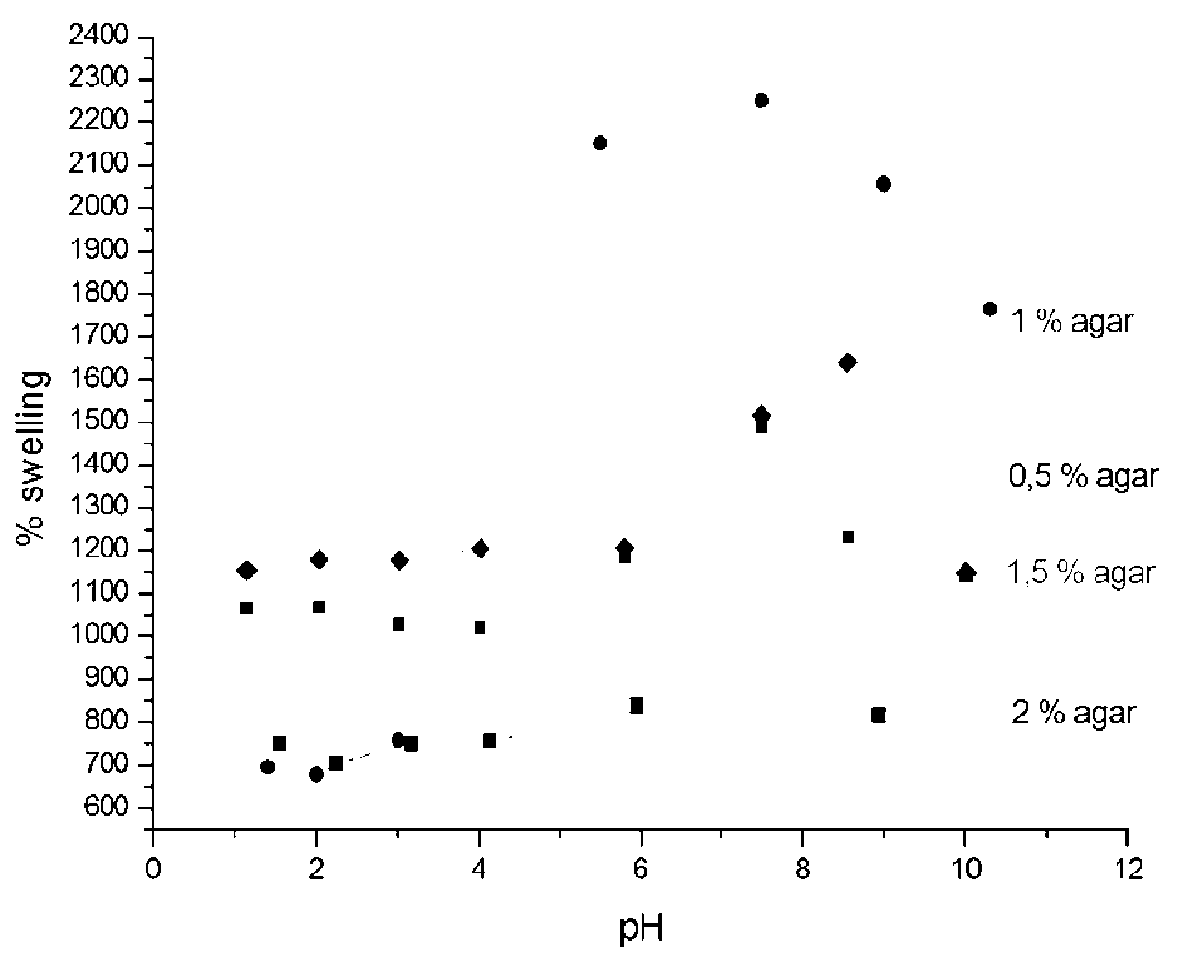

Fig. 3 The effect of pH (1-12) on the swelling behavior of the prepared hydrogels. polyhydroxy functional grouping which could increase hydrophilicity of the synthesized hydrogel.

If we increase the agar concentration beyond $1 \%$, a corresponding decrease in water retention is observed. We can say so, that when we add a higher amount of agar, crosslinking density decreases considerably and as a result, the network cannot hold enough water for swelling. Thus, increasing the percentage of agar was found to improve the swelling behavior of the hydrogel until $1 \%$ agar. In fact, due to the high crosslinking density of hydrogel with $0.5 \%$ agar, degree of swelling is low. This value is almost equal to hydrogel with $2 \%$ agar.

Beyond $1 \%$ of agar, there is no further increase in swelling capacity showing that hydrogel with $2 \%$ agar have a low crosslinking density and that its low crosslinking density may not be suitable to form a network. This may be explained by the fact that agar acts as a crosslinking inhibitor of PVP/PEG. And when the amount of agar increases, the crosslinking density decreases leading to a decrease in swelling degree. 
As a conclusion, since the SIPN is supposed to keep the agar fragment in place within the network, then, if the amount of agar is low, we are in the case of a high crosslinking density and water cannot penetrate the SIPN, leading to a decrease in swelling. And if agar proportion is high, crosslinking density decreases and the SIPN cannot hold enough agar in the network and agar can easily be removed leading to a decrease in swelling too.

Fig. 4 represents the hydrogel swelling behavior as a function of reaction time for samples containing 0.5 , $1,1.5$ and $2 \%$ of agar. At the first stage of the curves, the swelling rate is very high for $1 \%$ Agar, and the water can penetrate easily into the polymer network. The rest of hydrogel samples do not behave the same. For $0.5,1.5$ and $2 \%$ of agar it takes very much time to reach its swelling maximum. This can be explained by the crosslinking density of synthesized hydrogel.

In fact, if the crosslinking density is too high, the water cannot penetrate the SIPN so it takes very much time to reach equilibrium swelling, which is the case of the low percentage of agar. If the crosslinking density is too low, agar can be easily removed out of the network and we lose its polyhydroxy functional grouping what leads to a decrease in swelling and equilibrium takes place later.

$1 \%$ of agar is found to be the optimum percentage for crosslinking density. In fact, agar acts as a crosslinking inhibitor as illustrated in Abad works with Kappa-carrageenan, which have almost the same composition of agar except the sulfate functional grouping [23], whereby the PVP/PEG competes with agar for the free radicals. So, when the agar proportion increases, the crosslinking degree decrease, leading to a decrease in swelling.

A similar study of Jing et al. [24], using PDAMDMAC/Kappa-Carragenan hydrogel, has noted an increase in the water retention capacity when the amount of $\mathrm{KC}$ was increased from $0.5 \%$ to $1 \%$. And that no further increase was observed when $\mathrm{KC}$ was increased further.

As shown in Fig. 5, the relative rate of dehydration decreases with increasing the proportion of agar from 0.5 to $2 \%$, as a conclusion, when we add a higher amount of agar in the PVP/PEG blend, the kinetic become slower. As we demonstrated previously, beyond a percentage of $1 \%$ of agar, crosslinking density becomes lower and the presence of agar with his

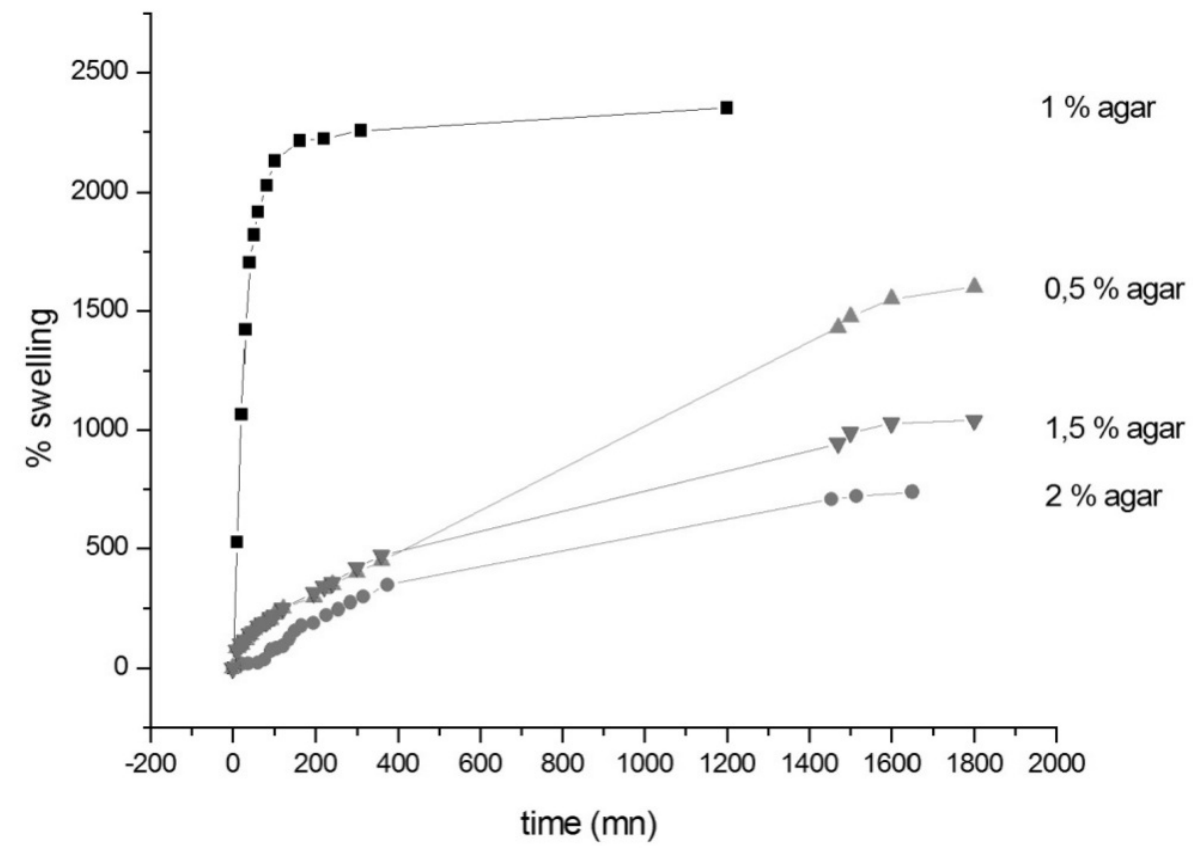

Fig. 4 The swelling \% of hydrogel as a function of contact time. 


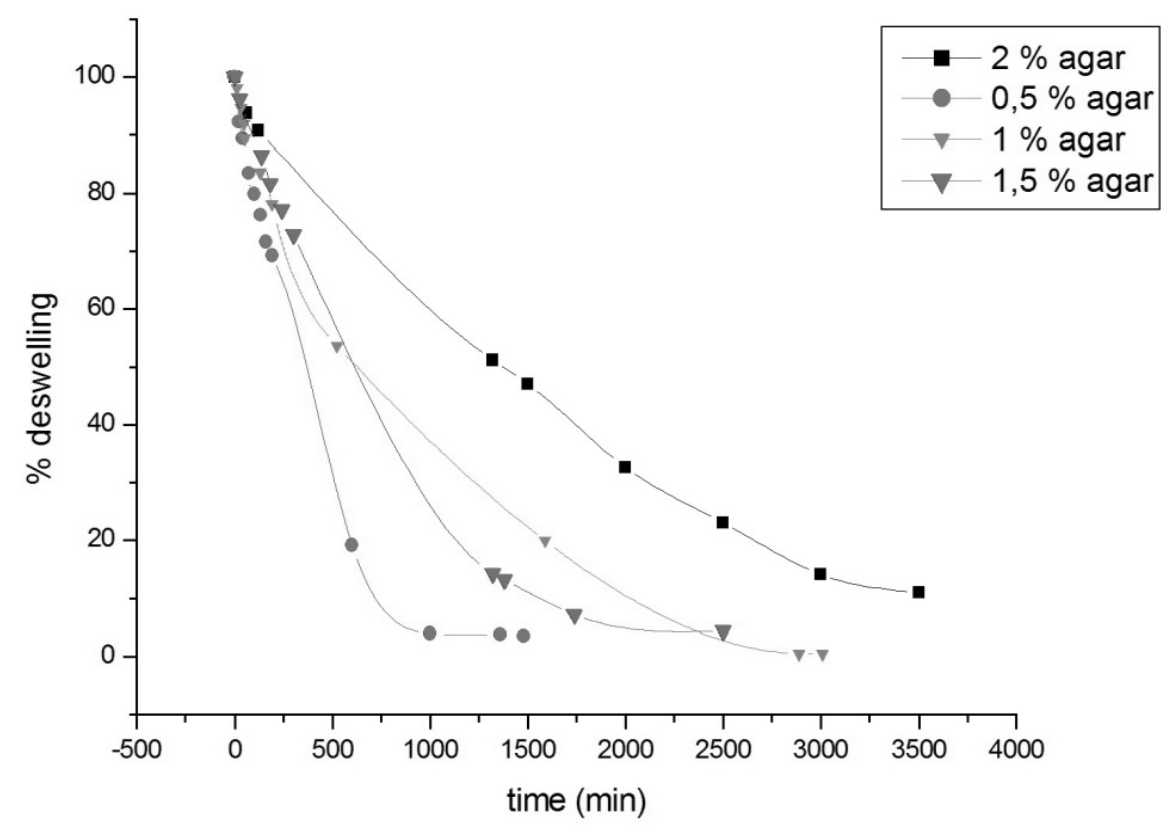

Fig. 5 The dehydration behavior isothermally as a function of time.

polyhydroxy functional grouping provides a better connection of the water in the polymer network. Otherwise, for low proportions of agar, the crosslinking density is higher [25], and the water is fixed essentially by entrapment in the network, what makes dehydration kinetic faster. But, if we increase the percentage of agar in the initial mixture, the contribution of polyhydroxy functional grouping is added to the entrapment process and this will improve the water retention in the network for longer time so water loss kinetic becomes slower.

\subsection{PH Dependence of Metal Retention}

All prepared hydrogel samples, were employed for removal of $\mathrm{Cu}$ (II) from aqueous solutions. Fig. 6 showed the adsorption capacities of 0.5 to $2 \%$ agar hydrogel for $\mathrm{Cu}(\mathrm{II})$ ions in solutions with initial concentration of $500 \mathrm{ppm}$ and varied $\mathrm{pH}$ from 1 to 5. At $\mathrm{pH}$ values higher than 5 , precipitation of $\mathrm{Cu}(\mathrm{II})$ ions to $\mathrm{Cu}(\mathrm{OH})_{2}$ occurred simultaneously, and could not lead to accurate interpretation of adsorption. According to Fig. 6, it was found that the hydrogel, including $1 \%$ of agar is able to fix $36.5 \mathrm{mg}$ of copper/g of dry gel but for $0.5 \%$ agar this decreases to $22.5 \mathrm{mg}$ of copper/g of dry gel. This can be explained by the easiness of access to sorption sites in contrast of the most crosslinked hydrogel containing $0.5 \%$ of agar. These results show that more the hydrogel develops a three dimensional network, more the adsorption capacities decreases. When the agar proportions exceed $1 \%$, crosslinking density decreases so that the network cannot hold enough water and cupper ions, and as we already demonstrated, agar acts as a crosslinking inhibitor.

It must be mentioned that since we act in acidic solutions, the adsorption capacities of hydrogels were low due to the presence of hydronium groups which induced an electrostatic repulsion of $\mathrm{Cu}$ (II) ions. Therefore, existing competition between hydronium and copper ions for adsorption sites resulted in the decrease of adsorption capacity $[11,26]$.

\subsection{Rate of Metal Ion Adsorption}

The kinetic study of adsorption is to establish the time course of $\mathrm{Cu}$ uptake on the hydrogel. It is also desirable to examine whether the behavior of $\mathrm{Cu}$ adsorption on the hydrogel can be described by a theoretical model that is predictive. The typical experimental results of adsorbed $\mathrm{Cu}$ on the hydrogel with $1 \%$ of agar versus time are shown in Fig. 7 since it 


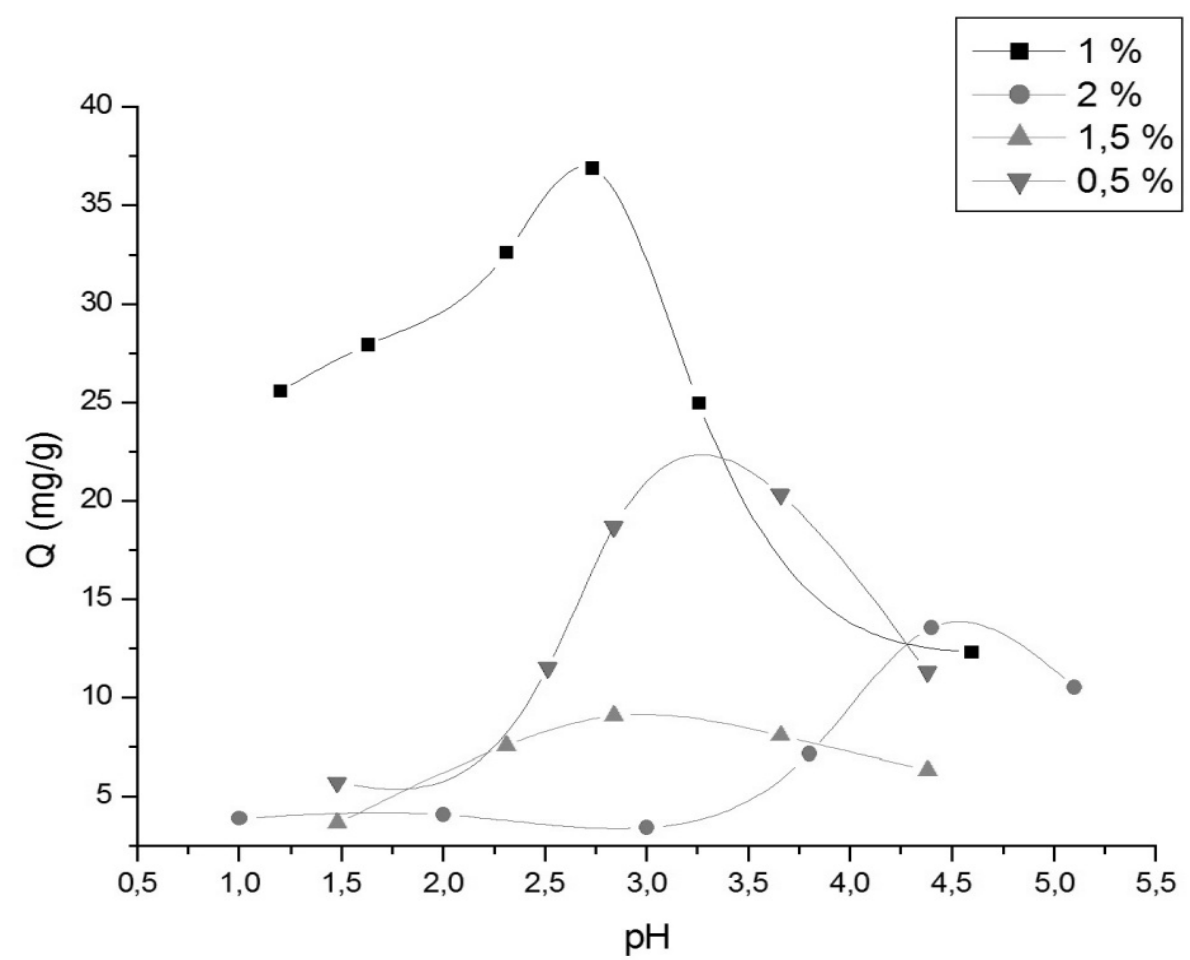

Fig. 6 The hydrogel adsorption capacities of $\mathrm{Cu}(\mathrm{II})$ ions in solutions with initial concentration of $500 \mathrm{mg} \cdot \mathrm{L}^{-1}$ and varied $\mathrm{pH}$ from 1 to 5.

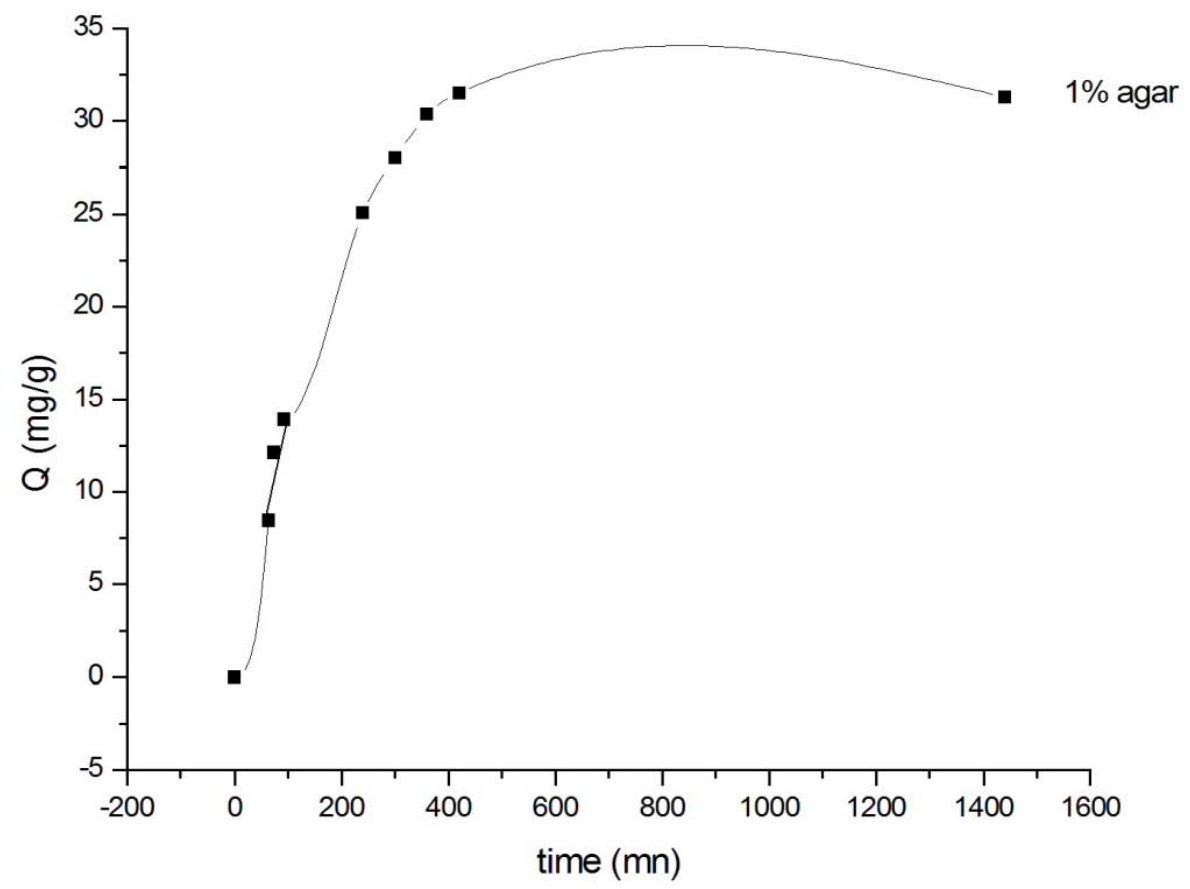

Fig. 7 copper II ion uptake by $1 \%$ agar hydrogel as a function of contact time.

shows a better retention capacity.

The adsorption equilibrium was reached at about $500 \mathrm{~min}$. Two steps of adsorption are considered: (i) the initial stage is the fast one and consists on the attachment of copper to the active adsorption sites on the hydrogel surfaces; (ii) the diffusion into inaccessible sites of adsorption in the hydrogel that takes much more time than the first step as illustrated 
Table 1 The kinetic parameters.

\begin{tabular}{lll}
\hline Kinetic model & \multicolumn{1}{c}{$1 \%$ Agar } \\
\cline { 2 - 3 } & $\mathbf{R}^{2}$ & Calculated parameters \\
\hline Pseudo first-order & 0.9848 & $\mathrm{~K}_{1}=0.0072 \mathrm{~min}^{-1}$ \\
Pseudo Second-order & 0.9889 & $\mathrm{~K}_{2}=9.5 \times 10^{-3}\left(\mathrm{~g} \cdot \mathrm{mg}^{-1} \mathrm{~min}^{-1}\right)$ \\
Elovich & 0.6755 & $\beta=1.2177(\mathrm{~g} \mathrm{mg})$ \\
Intraparticle Diffusion & 0.4148 & $\mathrm{~K}_{\text {int }}=0.1824$ \\
\hline
\end{tabular}

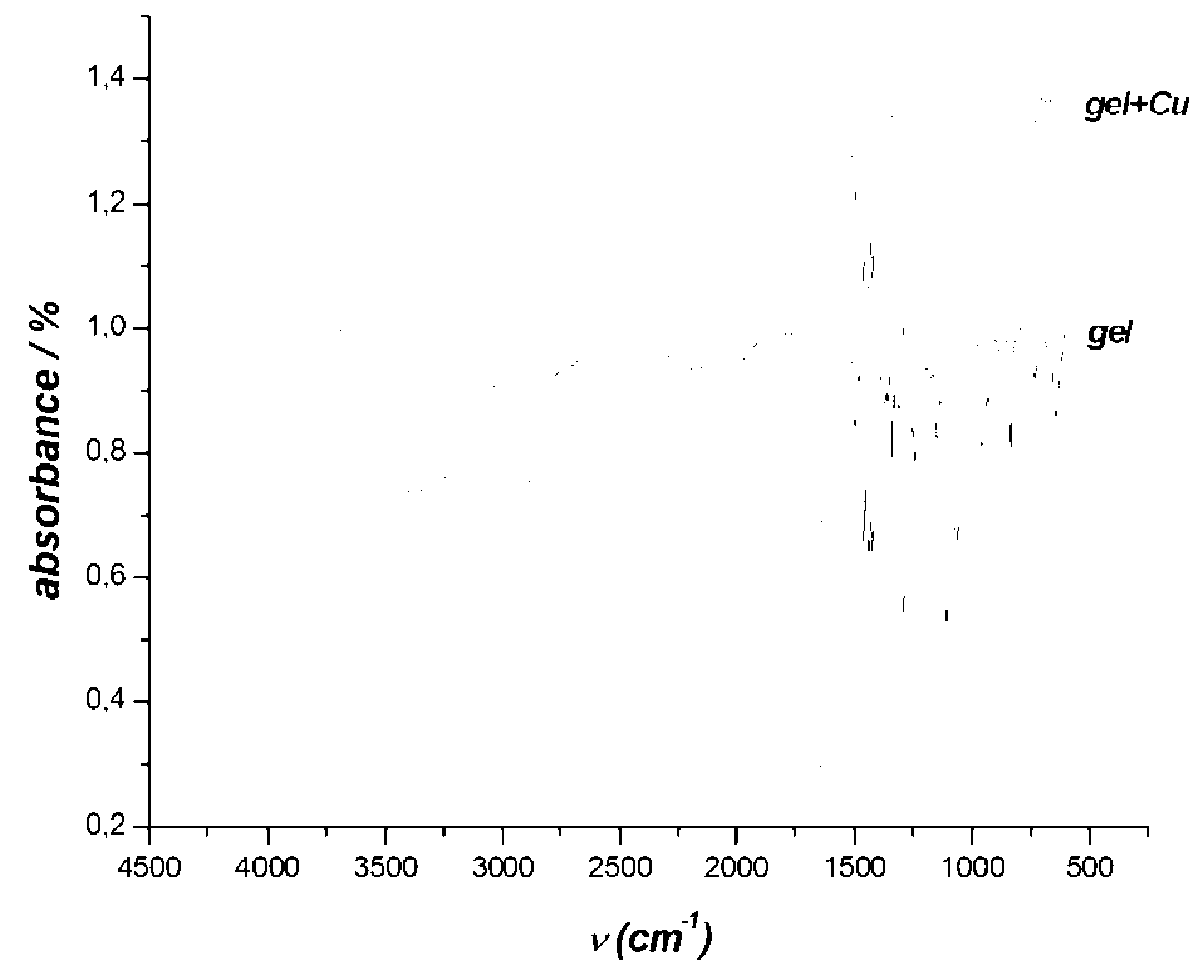

Fig. 8 FTIR spectra of PVP/PEG/Agar hydrogel (1\% of agar hydrogel irradiated at $50 \mathrm{kGy}$ ) before and after copper retention.

in Fig. 7. These results are in accordance with Nan Li and Renbi Bai works [27].

\subsection{Adsorption Kinetics}

The adsorption kinetic of the prepared hydrogel was undertaken with view of clarifying the controlling mechanism of copper adsorption process. This was visualized through kinetic models (see paragraph 2.7.3). $\operatorname{Ln}\left(q_{e}-q_{t}\right)$ vs. $t$ for first-order equation, $t / q$ vs. $t$ for a second-order equation, $q$ vs $\operatorname{Ln}(t)$ for Elovich model and $\mathrm{q}_{\mathrm{e}} \mathrm{vs}$. $\mathrm{t}^{(1 / 2)}$ for intraparticle diffusion model were plotted. The kinetic parameters of copper(II) ions onto hydrogel were calculated from these plots and are given in Table 1. It can be easily seen that the correlation coefficient $\mathrm{R}^{2}$ for pseudo first order and the Elovich kinetic models are lower than that of the pseudo-second order kinetic model. This result shows that the adsorption system is fitted to the pseudo-second-order kinetic model. The rate constant $\mathrm{k}$ of adsorption of ions can also be determined from the obtained straight line. Concerning the adsorption mechanism of the studied system, it is noted that there are two portions of the curve. The first portion is characterized by a considerable increase of the amount adsorbed $\mathrm{q}_{\mathrm{e}}$ depending on the square root of time, this is explained by the diffusion of copper in the macro and micro pores of the hydrogel. The second part of the curve is constant, attributed to the final equilibrium 
phase and that could possibly match the saturation of the adsorption sites.

\subsection{Copper Retention Verification Using FTIR Spectroscopy}

FTIR spectra of PVP/PEG/Agar hydrogel before and after copper retention are depicted in Fig. 8. It can be noticed that (i) the characteristic absorption band of polymeric $\mathrm{O}-\mathrm{H}$ at $830 \mathrm{~cm}^{-1}$ and $950 \mathrm{~cm}^{-1}$ (bending vibration) and also at $2,890 \mathrm{~cm}^{-1}$ (stretching vibration) were disappeared after reaction with copper (ii) Characteristic adsorption band at $1,645 \mathrm{~cm}^{-1}, 1,440$ $\mathrm{cm}^{-1}, 1,290 \mathrm{~cm}^{-1}, 1,100 \mathrm{~cm}^{-1}$, relative to $\mathrm{C}=\mathrm{O}, \mathrm{C}-\mathrm{N}$, and $\mathrm{C}-\mathrm{O}-\mathrm{C}$ stretching vibration respectively, persist showing they are not concerned with copper retention. As a conclusion, we can say that $\mathrm{Cu}^{2}+$ ions are immobilized by the hydrogel using Ionic connections which take place between $\mathrm{Cu}^{2+}$ and $\mathrm{C}-\mathrm{O}^{-}$.

\section{Conclusions}

$\mathrm{PVP} / \mathrm{PEG} /$ agar hydrogel is detected as an effective super absorbent polymer, the effect of changing agar proportion on the hydrogel characteristics was detected and showed that agar acts as a crosslinking inhibitor. In fact, PVP and PEG compete with agar for the free radicals. The incorporation of a higher amount of agar can improve the swelling behavior of hydrogel until $1 \%$ because agar contains the polyhydroxy functional grouping which could increase the hydrophilicity of the synthesized gel. This could be indicative of the presence of agar within the network either as an SIPN with permanent entanglement or as a grafted PVP and PEG.

The kinetic parameters were calculated from the experimental data and it was shown that they could be fitted well to the pseudo-second-order kinetic model. The mechanism of adsorption is explained by a diffusion of copper in the macro and micro pores of the hydrogel in the first step and then we reach the saturation of the adsorption sites. Finally, FTIR analysis clearly reveals that there have been interactions between PVP, PEG and Agar in order to constitute the superabsorbent polymer and that even copper is retained using Ionic connections which take place between $\mathrm{Cu}^{2+}$ and $\mathrm{C}-\mathrm{O}^{-}$.

\section{Acknowledgment}

The authors are grateful for scientific support from National Center of Sciences and Nuclear Technology and for the easiness of access to their equipments

\section{References}

[1] Pourjvadi, A., Ayyari, M. and Amini-Fazl, M. S. 2008. "Taguchi Optimized Synthesis of Collagen-g-Poly(Acrylic Acid)/Kaolin Composite Superabsorbent Hydrogel.” European Polymer Journal 44: 1209-16.

[2] Vogt, P., Roehlen, R. and Tennie, M. 2005. "Sealing Mat and Sealing Web Comprising a Superabsorbent Layer, Method for the Production Thereof, and Use Thereof." European Polymer Patent. 1: 534-15.

[3] Stradeling, M. A. and Goene, F. 2001. "Powder Free Super Absorbent Tapes and Yarns for Optical Fiber Cables." Process International Wire Cable Symposium 50: 526.

[4] Karadag, E., Saraydin, D., Caldiran, Y., Guven, O. 2000. "Swelling Studies of Copolymeric Acrylamide/Crotonic Acid Hydrogels as Carriers for Agricultural Uses." Polymers for Advanced Technologies 11: 59-68.

[5] Sadeghi, M., Hosseinzadeh, H. J. 2008. "Synthesis of Starch-Poly (Sodium Acrylateco-Acrylamide) Superabsorbent Hydrogel with Salt and PH-Responsiveness Properties as a Drug Delivery System". Journal of Bioactive and Compatible Polymers 23: 381-404.

[6] Ajji, Z., Mirjalili, G., Alkhatab, A. and Dada, H. 2008. "Use of Electron Beam for the Production of Hydrogel Dressings." Radiation Physics and Chemistry 77: 200-02.

[7] Kas-goz, H., Durmus, A. and Kas-goz, A. 2008. "Enhanced Swelling and Adsorption Properties of AAm-AMPSNa/Clay Hydrogel Nanocomposites for Heavy Metal Ion Removal." Polymers for Advanced Technologies 19: 213-20.

[8] Rosiak, J. M., Olejniczak, J. and Pekala, W. 1990. "Fast Reaction of Irradiated Polymers Crosslinking and Degradation of Polyvinylpyrrolidone." Radiation Physics and Chemistry 36: 747-55.

[9] Lilian, C., Lopérgolo, B., Ademar, L. and Catalani, H. 2003. "Direct UV Photocrosslinking of Poly (N-Vinyl-2-Pyrrolidone) (PVP) to Produce Hydrogels." 
Polymer 44: 6217-22.

[10] Farah, K., Jerbi, T., Kuntz, F. and Kovacs, A. 2006. "Dose Measurements for Characterization of a Semi-Industrial Cobalt-60 Gamma-Irradiation Facility." Radiation Measurement 41: 201-8.

[11] Dai, J., Yan, H., Yang, H. and Cheng, R. 2010. "Simple Method for Preparation of Chitosan/Poly(Acrylic Acid) Blending Hydrogel Beads and Adsorption of Copper(II) from Aqueous Solutions." Chemical Engineering Journal 165: 240-9.

[12] Bangash, F. K. and Alam, S. 2009. "Adsorption of Acid Blue 1 on Activated Carbon Produced from the Wood of Ailanthus Altissima." Brazilian Journal of Chemical Engineering 26: 2.

[13] Ho, Y. S. and McKay, G. 1998. "Kinetic Models for the Sorption of Dye from Aqueous Solution by Wood." Journal of Environment Science Health Part B: Process Safety Environment Protect 76: 183-91.

[14] Low, M. J. D. 1960. "Kinetics of Chemisorption of Gases on Solids." Chemical Review 60: 267-312.

[15] Ponnusami ,V., Vikram, S. and Srivastava, S. N. 2008. "Guava (Psidium Guajava) Leaf Powder: Novel Adsorbent for Removal of Methylene Blue from Aqueous Solutions." Journal of Hazardous Material 152: 276-86.

[16] Bardajee, G. R., Pourjavadi, A., Sheikh, N. and Amini-Fazl, M. S. 2008. "Grafting of Acrylamide onto Kappa-Carrageenan via g-Irradiation: Optimization and Swelling Behavior." Radiation Physics and Chemistry 77: 131-7.

[17] Davis, J. E., Sangster, D. F. and Senogles, E. 1981. "Pulse Radiolysis of Aqueous Solutions of N-vinylpyrrolidin-2-one and Poly(N-vinylpyrrolidin-2-one)". Australian Journal of Chemistry 34: 1423-31.

[18] Abad, L., Revelle, V., Lorna S., Aranilla T., Dela, R. and Alumanda, M. 2003. "Properties of Radiation Synthesized PVP-Kappa Carrageenan Hydrogel Blends." Radiation Physics and Chemistry 68: 901-08.

[19] Roy, N., Saha, N., Kitano, T. and Saha, P. 2012.
"Biodegradation of PVP-CMC Hydrogel Film: A Useful Food Packaging Material." Carbohydrate Polymers 89: 346-53.

[20] Wang, W. and Wang, A. 2010. "Synthesis and Swelling Properties of PH-Sensitive Semi-IPN Superabsorbent Hydrogels Based on Sodium Alginate-g-Poly (Sodium Acrylate) and Polyvinylpyrrolidone." Carbohydrate Polymers 80: 1028-36.

[21] Lugao, A. B. and Malmonge, S. M. 2001. "Use of Radiation in the Production of Hydrogels." Nuclear Instruments and Methods in Physics Research Section B: Beam Interactions with Materials and Atoms 185: 37-42.

[22] Bin Ahmad, M., Tay, M. Y., Shameli, K., Hussein, M. Z. and Lim, J. J. 2011. "Green Synthesis and Characterization of Silver/Chitosan/Polyethylene Glycol Nanocomposites without any Reducing Agent." International Journal of Molecular Science 12: 4872-84.

[23] Abad, L., San Diego, C., Relleve, L., Aranilla, C., dela Rosa, A. M. and Janik, I. et al. 1999. "Sol-Gel Analysis of Crosslinked Polymers." In Proceedings 15th Philippine Chemistry Congress, Cebu City, Philippines, 466.

[24] Jing, R., Yanqun, Z., Jiuqiang, L. and Hongfei, H. 2001. "Radiation Synthesis and Characteristic of IPN Hydrogels Composed of Poly(Diallyldimethylammonium Chloride) and Kappa-Carrageenan." Radiation Physics and Chemistry 62: 277-81.

[25] Ajji, Z., Othman, I. and Rosiak, J. M. 2005. "Production of Hydrogel Wound Dressings Using Gamma Radiation." Nuclear Instruments and Methods in Physics Research, Beam Interactions with Materials and Atoms 229: 375-80.

[26] Özer, A., Özer, D. and Özer, A. 2004. "The Adsorption of Copper(II) Ions on to Dehydrated Wheat Bran (DWB): Determination of the Equilibrium and Thermodynamic Parameters." Process Biochemistry 39: 2183-91.

[27] Li, N. and Bai, R. 2005. "Copper Adsorption on Chitosan-Cellulose Hydrogel Beads: Behaviors and Mechanisms". Separation and Purification Technology 42: $237-47$. 